ARTICLE

\title{
Analysis of erythrocyte signalling pathways during Plasmodium falciparum infection identifies targets for host-directed antimalarial intervention
}

Jack D. Adderley (10 1, Simona John von Freyend (10 2, Sarah A. Jackson², Megan J. Bird ${ }^{2}$, Amy L. Burns ${ }^{3}$, Burcu Anar ${ }^{4}$, Tom Metcalf ${ }^{4}$, Jean-Philippe Semblat ${ }^{5}$, Oliver Billker (D) ${ }^{4,6}$, Danny W. Wilson (1) ${ }^{3,7} \&$ Christian Doerig (iD ${ }^{1 \times}$

Intracellular pathogens mobilize host signaling pathways of their host cell to promote their own survival. Evidence is emerging that signal transduction elements are activated in anucleated erythrocytes in response to infection with malaria parasites, but the extent of this phenomenon remains unknown. Here, we fill this knowledge gap through a comprehensive and dynamic assessment of host erythrocyte signaling during infection with Plasmodium falciparum. We used arrays of 878 antibodies directed against human signaling proteins to interrogate the activation status of host erythrocyte phospho-signaling pathways at three blood stages of parasite asexual development. This analysis reveals a dynamic modulation of many host signalling proteins across parasite development. Here we focus on the hepatocyte growth factor receptor (c-MET) and the MAP kinase pathway component B-Raf, providing a proof of concept that human signaling kinases identified as activated by malaria infection represent attractive targets for antimalarial intervention.

\footnotetext{
${ }^{1}$ Centre for Chronic Inflammatory and Infectious and Diseases, Biomedical Sciences Cluster, School of Health and Biomedical Sciences, RMIT University, Bundoora, VIC 3083, Australia. ${ }^{2}$ Infection and Immunity Program, Department of Microbiology, Biomedicine Discovery Institute, Monash University, Clayton, VIC 3800, Australia. ${ }^{3}$ Research Centre for Infectious Diseases, School of Biological Sciences, University of Adelaide, Adelaide, SA 5005, Australia. ${ }^{4}$ Wellcome Trust Sanger Institute, Hinxton, Cambridgeshire CB10 1SA, UK. ${ }^{5}$ Institut National de la Transfusion Sanguine, Inserm UMR S1134, 75015 Paris, France. ${ }^{6}$ Molecular Infection Medicine Sweden (MIMS), Department of Molecular Biology, Umeå University, Umeå SE-901 87, Sweden. ${ }^{7}$ Burnet Institute, Melbourne, VIC 3004, Australia. ${ }^{凶}$ email: christian.doerig@rmit.edu.au
} 
M alaria, caused by infection of mosquito-borne apicomplexan parasites in the genus Plasmodium, remains one of the most devastating infectious diseases globally. Among the six species that infect humans, Plasmodium falciparum is the most virulent and is responsible for the majority of malaria-related deaths. The remaining human malaria species Plasmodium vivax, Plasmodium ovale, Plasmodium malariae and two zoonotic species, Plasmodium knowlesi and Plasmodium simium $^{1,2}$, are major contributors to global malaria morbidity and must be considered in the context of new treatment strategies for malaria. Recent years have seen a 50\% drop in malaria-related mortality, yet the disease still kills an estimated 445,000 people every year, mostly young children in sub-Saharan Africa ${ }^{3}$. Progress towards malaria elimination and ultimately eradication has stagnated, and the emergence of parasites resistant to the most recently deployed global frontline treatment, artemisinincombination therapies ${ }^{3,4}$, is a major concern. It remains critical to develop novel antimalarial drugs with untapped modes of action.

Protein kinases (PKs) are core components of signalling pathways in eukaryotic cells. Phosphorylation of the target protein can lead to conformational changes and generate or mask binding motifs, and thus can affect its activity, binding properties, stability or subcellular localization. PK activity can be regulated positively or negatively by phosphorylation of specific amino acids, or by binding to specific activator or inhibitor proteins. Activation leads to a conformational change making the active site accessible to ATP and the substrate protein. PKs are eminently druggable targets, as illustrated by the fact that $>48$ small molecule kinase inhibitors have reached the market, mostly in the context of cancer chemotherapy $y^{5,6}$. The kinomes of apicomplexan parasites and humans are quite divergent, owing to the large phylogenetic distance between these organisms ${ }^{7}$, and parasiteencoded PKs have been proposed as attractive potential targets for selective intervention ${ }^{8}$. Host-directed therapy (HDT) is another avenue that is currently gaining traction to combat infectious diseases generally ${ }^{9}$. We and others ${ }^{10,11}$ have proposed that host erythrocyte (and hepatocyte) PKs represent excellent potential targets for antimalarial intervention. Many antiinfective drugs are rendered ineffective by the selection of mutations in their pathogen-encoded targets, which explains the rapid emergence of resistance across essentially all pathogen taxons. Targeting host enzymes would deprive the pathogen of this most direct pathway to resistance. Furthermore, repurposing anti-cancer kinase inhibitors as agents against infectious diseases would greatly alleviate the lack of resources that severely affects anti-infective drug development.

Malaria pathology is caused by asexual proliferation of the parasite in erythrocytes. Blood-stage malaria begins with the infection of erythrocytes by Plasmodium merozoites, followed by rapid growth and asexual multiplication (schizogony). Newly formed merozoites are then released into the bloodstream and invade erythrocytes to begin the next replication cycle $(\sim 48 \mathrm{~h}$ for $P$. falciparum). Several human signalling molecules of the host erythrocyte have been implicated in parasite survival during infection. These include G-coupled protein receptors ${ }^{12-14}$, the PKs MAP/ERK kinase $(\mathrm{MEK})^{15}$, p21-activated kinase $(\mathrm{PAK})^{15}$, Protein kinase $C(\mathrm{PKC})^{16}$, and peroxiredoxin ${ }^{17-19}$. In our study of the erythrocyte PAK-MEK pathway ${ }^{15}$, we showed that host MEK1 is phosphorylated on residue Ser298 in infected erythrocytes. pSer298 is known to cause activation of the enzyme through stimulating trans-autophosphorylation on its activation loop. The only PK known to phosphorylate MEK1 on Ser298 is PAK1 ${ }^{20}$ and, indeed, we showed that the latter enzyme is also activated in infected erythrocytes ${ }^{15}$. PAK isoforms are notorious for having a multitude of substrates ${ }^{21}$ and serve as a node to integrate signals from a number of upstream receptors and transmit these signals to several downstream effector pathways (including the mitogen-activated PK pathway (MAPK), which includes the aforementioned MEK). Therefore, we hypothesized that our description of the PAK-MEK pathway addresses but a minute fraction of the signalling events that may be modulated by infection. Here, to comprehensively assess the host erythrocyte signalling response to infection, we use a microarray of antibodies designed to recognize human signalling proteins and their phosphorylation status. Comparison of the signals obtained from extracts of uninfected erythrocytes with those obtained from erythrocytes infected with P. falciparum at three main developmental stages of the replication cycle (rings, trophozoites and schizonts) allowed us to generate a comprehensive and dynamic picture of the modulation of host erythrocyte signalling by infection. This identified several host kinases as potential targets for HDT; on this basis, we further demonstrate that selective inhibitors against human c-MET and B-Raf display high potency against $P$. falciparum and $P$. knowlesi in vitro, and show that a cMET inhibitor has in vivo activity against Plasmodium berghei in a murine model of malaria.

\section{Results}

Kinexus antibody microarray analysis. To investigate dynamic changes in host erythrocyte signalling during $P$. falciparum asexual proliferation, we employed an antibody microarray developed by Kinexus (Vancouver, Canada). The array consists of 878 unique antibodies, 265 of which are pan-specific, i.e., recognize both the phosphorylated and unphosphorylated forms of the target protein; the remaining 613 antibodies are phosphorylation-specific, recognizing signalling molecules only if their activating or inhibitory phosphorylation sites are modified by the addition of a phosphate group. Some of the most important and well-known signalling molecules, such as members of the MAPK pathways or the PKC isoforms, are detected by multiple antibodies aimed at various phosphorylation sites within the same protein. The array thus provides a comprehensive picture as to how signalling mediated by these molecules changes during infection. Each array device comprises two identical chambers, each carrying two spots for each of the 878 antibodies, thus delivering each read-out in duplicate. Two sample lysates are labelled with protein-binding fluorescent dye (e.g., from Plasmodium-infected (iRBC) and non-infected ( $\mathrm{URBC}$ ) red blood cells) and compared on each device, one sample per chamber. Binding of proteins to the antibodies on the array is quantitated through signal intensity for each spot.

Robustness of the approach. To evaluate the reproducibility and robustness of the approach, we compared four uninfected erythrocyte (uRBC) samples from different blood donors that were cultured for $\sim 24 \mathrm{~h}$ under the same conditions as the iRBCs. The mean signal intensity and SD for each phospho-specific antibody on the array were determined for these four samples (Fig. 1; the entire datasets are available in Supplementary Data 1). Despite some variation, individual data points for each antibody remained within $2 \mathrm{SDs}$ of their mean, illustrating that sample-to-sample variation was minimal.

Overall changes in phosphosignaling during infection. To assess the impact of infection on host cell signalling, whole-cell protein extracts were obtained from synchronized $P$. falciparum cultures at three time windows during the erythrocytic cycle: 4-12 h post invasion (hpi) (rings, $n=3$ ), 24-28 hpi (trophozoites, $n=3$ ) and 44-48 hpi (segmented schizonts, $n=2$ ). Each of these extracts were loaded onto a separate antibody array and 


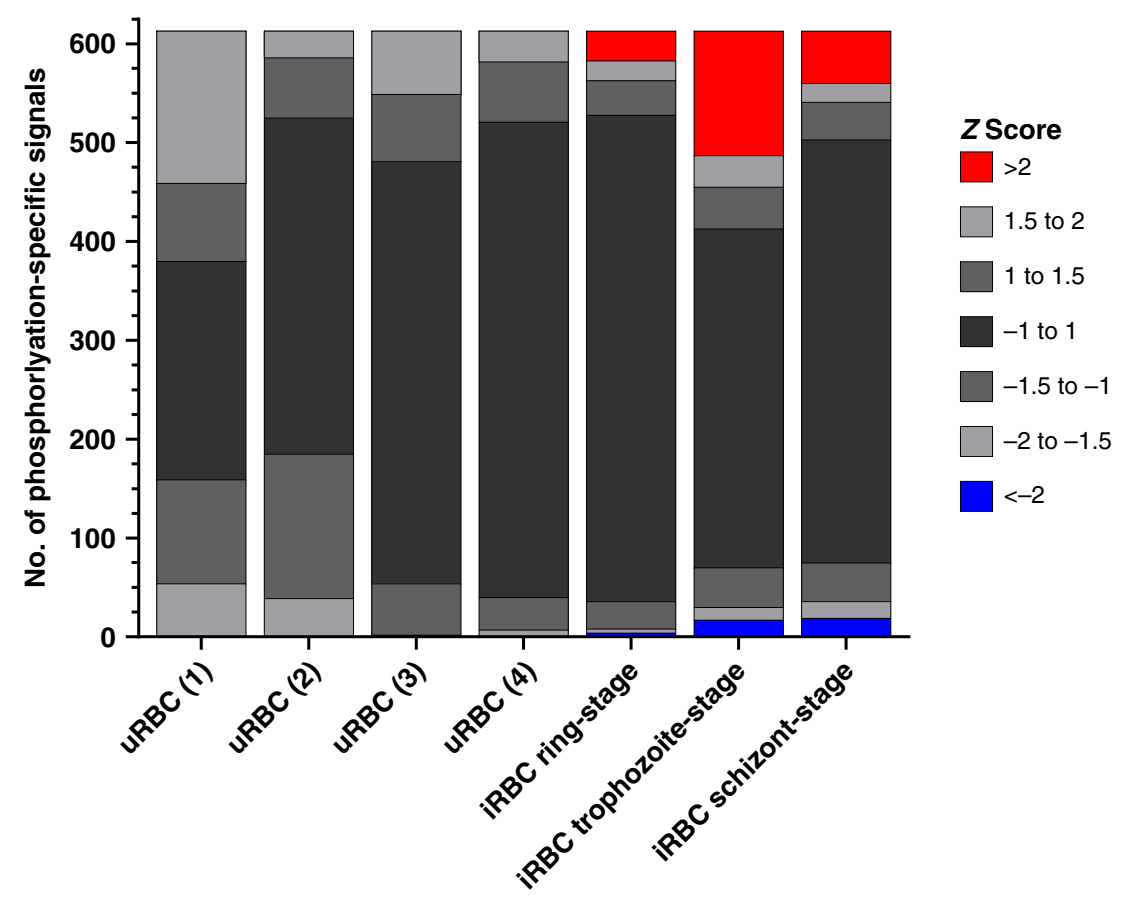

Fig. 1 Distribution of Z-scores for each sample. All antibody microarray signals from each sample (four from uRBCs and the mean signal for each iRBC harbouring parasites at the ring $(n=3)$, trophozoite $(n=3)$ and schizont stage $(n=2))$ were compared with the uninfected erythrocyte mean value for each signal, and the Z-score determined for each phosphorylation-specific signal. The Z-score is defined as the number of SDs from the uRBC mean for each sample; a positive or negative value indicates the direction of the signal (increase or decrease, respectively) from the uRBC mean. No signals from the uRBC samples $(n=4)$ had associated Z-scores above 2 or below -2 , whereas the three iRBC time points showed clear variations from uRBCs. Source data are provided as a Source Data file.

compared to the mean of the uRBC lysates collected in parallel and containing RBCs from the same donor as the iRBC sample (see above). In stark contrast to the minimal variation between the uRBC samples (see above), analysis of the iRBC extracts showed that $6 \%$ (ring-stage), 23\% (trophozoite-stage) and $12 \%$ (schizont stage) of the phospho-specific antibody intensities differed by more than 2 SDs from uRBC mean. The datasets for each of the three developmental stages showed no internal variation $>2$ SDs between the biological replicates (Supplementary Fig. 1), further confirming the robustness and reliability of the approach (Fig. 1; the complete datasets are available in Supplementary Data 1). Overall, this implies (i) that there is little variation between biological replicates of uRBCs, attesting to the reliability of the system, and (ii) that infection with $P$. falciparum causes significant variation in the signals yielded by phospho-specific antibodies, suggesting that $P$. falciparum strongly impacts host erythrocyte phosphosignalling during infection.

Data filtering: removal of cross-reacting antibodies. To address possible cross-reactivity of the antibodies with parasite-derived proteins, we compared signals from purified unsynchronized parasites (pellet obtained by saponin lysis followed by centrifugation) with those of the purified erythrocyte cytoplasm (saponin supernatant). Saponin disrupts the erythrocyte membrane, thereby releasing erythrocyte cytoplasmic proteins and exported parasite proteins, while the parasite and insoluble erythrocytic material can be pelleted 22 . The array was loaded with $20 \times$ more (protein mass) pellet material than supernatant material, to confer high stringency to the cross-reactivity filter. A heatmap of the results is shown in Supplementary Fig. 2a (full data available in Supplementary Data 2). Antibodies showing a fold change $>1.5$ between the erythrocyte cytoplasm (saponin supernatant) and the parasite extract (saponin pellet), amounting to 224 signals $(37 \%)$ of the phospho-specific antibodies, were withdrawn from further analysis (Supplementary Fig. 2 and Supplementary Data 2). This level of cross-reactivity is not surprising, as many signalling proteins display conservation between Homo sapiens and P. falciparum, especially in their regulatory sites ${ }^{7}$. Some of these cross-reactive antibodies may recognize parasite-encoded orthologues of the human target proteins and thus prove to be useful tools to study $P$. falciparum signal transduction; however, this lies outside of the scope of the present study.

Data filtering: removal of low-signal antibodies. Some antibodies displayed a weak fluorescence signal, likely due to the low abundance of the target protein. The antibodies yielding a signal intensity below a fluorescence reading of 1000 units in both the erythrocyte control and parasite-infected samples were removed from further analysis, as recommended by Kinexus (Supplementary Data 1). This included 26 signals from the ring array, and 41 and 48 signals from the trophozoite and schizont arrays, respectively.

Broad analysis of the post-filtering iRBC data set. Following low and cross-reactive signal removal, 1 of the ring-stage signals, 29 of the trophozoite-stage and 17 of the schizont-stage signals had fold changes $>2$ or $<0.5$ compared to their uninfected counterpart, revealing dynamic changes in the phosphorylation of host signalling proteins during $P$. falciparum asexual development (Fig. 2a: heatmap of all retained signals; Fig. 2b: distribution of increased and decreased signals at the three development stages). The small number of changes in ring-infected cells may in part be due to the fact that these samples contained only $33 \%$ infected cells (as magnetic purification of infected cells, which allows close to $100 \%$ iRBCs for the trophozoite and schizont stages, cannot be 
a

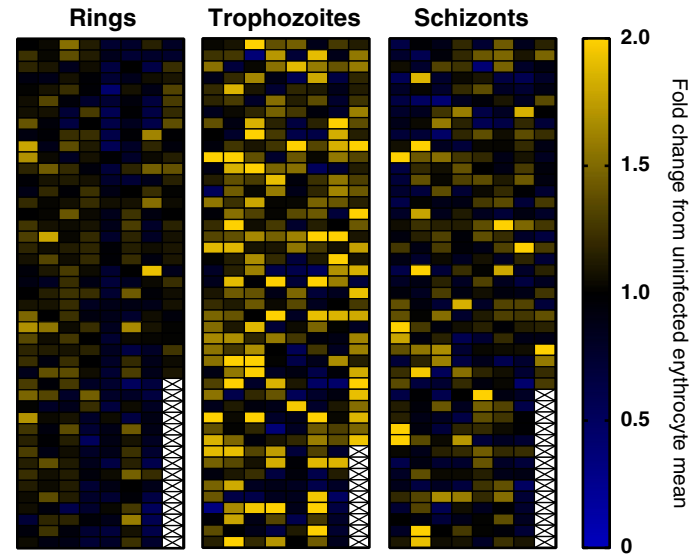

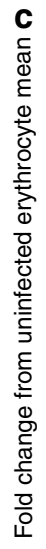
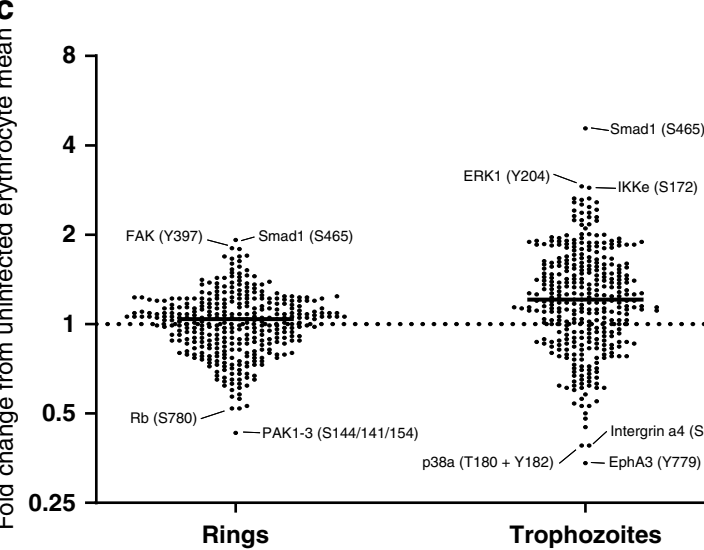

Trophozoites

b
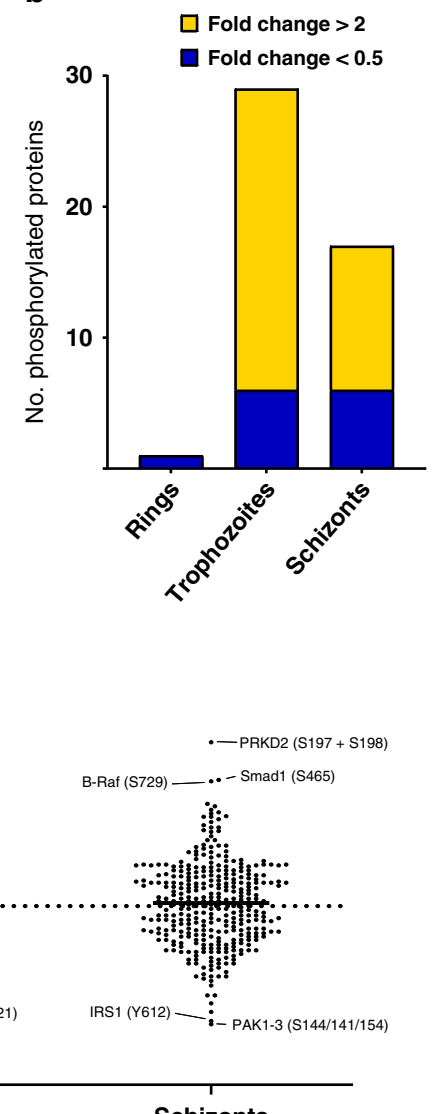

Schizonts

Fig. 2 Microarray data for the ring, trophozoite and schizont stages. a Heatmap of phosphospecific antibody array data for ring-, trophozoite- and schizont-infected cells after the removal of cross-reactive and weak signals. b Phosphorylation-specific signals (post-filtering) ranked according to fold change. Yellow represents changes with a fold change $>2$; blue represents changes with a fold change $<0.5$. Full datasets are presented in Supplementary Data 1 and 2. c Dot plot highlighting differences in phosphosite signals represented as fold change. The largest changes are listed with their associated phosphorylation site. Source data are provided as a Source Data file.

implemented for ring stages). The observed effect on host cell phosphosignaling was considerably larger at later stages of infection (trophozoite/schizont), with the majority of these changes being attributed to increases in phosphorylation $(80 \%$ and $65 \%$, respectively). A dot plot for each of the three life stages (Fig. 2c) further illustrates this trend and highlights the strongest individual changes.

Shortlisting of significant changes in phosphosignaling. Of the reliable antibodies remaining after removal of low and crossreactive signals, 16/345 (ring stage), 102/351 (trophozoite stage) and $35 / 346$ (schizont stage) were significantly different $(p<0.5$, unpaired $t$-test) from the uninfected erythrocyte signals. Figure 3 lists the most noteworthy changes observed from this analysis (the full list of the significant changes is available in Supplementary Data 3).

Some of the most notable changes implicated the PKC isoforms $\delta$ and $\theta$. Several PKC isoforms have previously been reported to be activated during $P$. falciparum infection of erythrocytes, but the phosphorylation status of these kinases was uncharacterized ${ }^{23,24}$. Several phosphorylation sites of PKC 8 / $\theta$ were significantly phosphorylated in trophozoite- and schizontinfected cells (Fig. 3; a list of the phosphorylation-specific signals for $\mathrm{PKC} / \theta$ is available in Supplementary Fig. 3). The most striking changes were observed for Thr507 (PKC $\delta$ ) and Ser695 $(\mathrm{PKC} \theta)$. Thr507 is a regulatory residue located within the activation loop of $\mathrm{PKC} \delta^{25}$, whereas Ser695 is within the hydrophobic motif of $\mathrm{PKC} \theta$, and its phosphorylation is required for optimal kinase activity ${ }^{26}$. In addition, phosphorylation of Ser695 may also be involved in the localization of PKC $\theta$ to the plasma membrane $\mathrm{e}^{27}$. These data are consistent with previous reports that host cell PKC activity is detectable during schizont development (30-40 h post invasion) with limited activity before $25 \mathrm{~h}^{23,24}$, and considerably refine these previous findings, notably with respect to dynamic phosphorylation changes on specific residues of the various $\mathrm{PKC}$ isoforms during parasite development.

MAP kinase signalling in infected erythrocytes. The canonical MAPK pathway is a highly conserved, three-tiered cascade, in which a MAP kinase kinase kinase (MAP3K, or MEKK for MAP/ ERK kinase kinase) phosphorylates and thereby activates a MAP2K (or MEK), which in turn phosphorylates and activates a MAP kinase. A well-understood MAPK pathway involves Raf, MEK and ERK. Both Raf isoforms are activated by Ras-GTPase binding, which enables phosphorylation of T598 and S601 (on BRaf), and S338 and Y340 (on C-Raf) ${ }^{28-30}$. This results in the full activation of Raf, enabling downstream phosphorylation of MEK and, consequently, ERK1/2 (pathway depicted in Fig. 4b). $P$. falciparum has no homologues for either Ras, Raf or MEK, but expresses two MAPKs, neither of which is a close homologue of ERK $1 / 2^{31}$. Figure 4 lists the phosphorylation sites detected on the array that are relevant to canonical MAPK pathways (Fig. 4a) and 


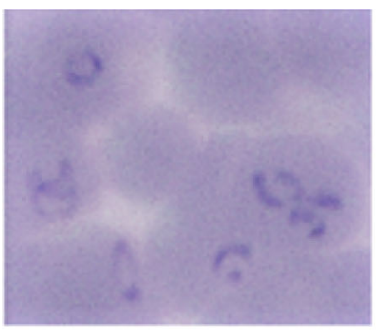

Rings

\begin{tabular}{|c|c|c|c|}
\hline Protein & $\begin{array}{c}\text { Phospho site } \\
\text { (human) }\end{array}$ & $\begin{array}{c}\text { Fold } \\
\text { change }\end{array}$ & $\boldsymbol{p}$ value \\
\hline FAK & Y397 & 1.80 & 0.043 \\
\hline DDR1 & Y796 + Y797 & 1.69 & 0.027 \\
\hline B-Raf & S729 & 1.69 & 0.029 \\
\hline SCYL1 & S754 & 1.63 & 0.045 \\
\hline PTEN & S380 + T382+ T383 & 1.43 & 0.042 \\
\hline HSP90AB1 & Y484 & 1.38 & 0.044 \\
\hline DDR2 & Y736 & 1.34 & 0.044 \\
\hline CHK1 & S317 & 1.30 & 0.019 \\
\hline JAK2 & Y570 & 1.20 & 0.044 \\
\hline Rac1/cdc42 & S71 & 0.65 & 0.010 \\
\hline RSK1/2 & S380/S386 & 0.64 & 0.006 \\
\hline Tau & S713 & 0.58 & 0.050 \\
\hline Paxillin 1 & Y31 & 0.56 & 0.012 \\
\hline Tau & S713 & 0.53 & 0.011 \\
\hline p38a MAPK & T180 + Y182 & 0.52 & 0.045 \\
\hline Rb & S780 & 0.52 & 0.029 \\
\hline
\end{tabular}

Fig. 3 Shortlist of the highest confidence signals $(\boldsymbol{p} \mathbf{0 . 0 5})$ for rings, trophozoites and schizonts. Increases in phosphorylation compared to uninfected erythrocytes are marked in yellow; decreases are marked in blue. All values listed are the fold change from the uRBC control mean ( $n=4$ biologically independent samples); the corresponding $p$-value for each signal is also listed. Each signal has a $p$-value $<0.05$, as determined through an unpaired twotailed T-test. All listed signals passed stringent parasite cross-reactivity and signal intensity filters (see text for details and Fig. 2 ).

indicates the activating phosphorylation sites for each of the three kinases involved (Fig. 4b).

Proteins in the MAPK signalling pathway showed notable changes in phosphorylation in iRBCs compared with uRBCs. BRaf and C-Raf as the 'gatekeeper' proteins of this pathway showed changes in phosphorylation patterns with $P$. falciparum $\mathrm{RBC}$ infection. Unfortunately, the activating Ras-induced phosphorylation sites on B-Raf (T598 and S601) and C-Raf (S338 and Y340) are not represented in the array and could not be assessed. Antibodies against C-Raf S296 yielded a consistent fold change of $>1.2$ for all three parasite stages. Phosphorylation of C-Raf on S296 is thought to be mediated by ERK1/2 in the context of a negative feedback loop ${ }^{32}$, which would be consistent with activation of the MAPK pathway detected here (see below).

B-Raf phosphorylation at S446 and S447, sites associated with priming B-Raf for activation by Ras ${ }^{30,33}$, increased by 1.79 -fold at the ring stages, but decreased back to uRBC levels in trophozoites and schizonts. B-Raf S446 is constitutively phosphorylated in some cell lines, but this remains to be shown in cells of the erythropoietic lineage $e^{30,33-35}$. In contrast, phosphorylation of BRaf at S729, a Ras-independent phosphorylation event carried out by AMP-activated PK (AMPK) in response to energy stress, increased at all three life cycle stages with a fold change ranging from 1.69 to 2.63 across parasite development (Figs. 3 and $4 \mathrm{a}$ ). Of the two AMPK antibodies on the array, one was discounted due to cross-reactivity with a parasite protein, whereas the other (AMPKa2 S377) shows a slight increase (1.17-fold change) in phosphorylation at the trophozoite time point, supporting that this kinase may also be activated in iRBCs (Supplementary Data 1).

We next determined the total amount of B-Raf and phosphorylated B-Raf S729 at each life cycle stage using western blotting. Detection of total B-Raf protein using a pan-antibody independent of phosphorylation showed that the amount of fulllength B-Raf was greatly reduced at trophozoite and schizont stages compared to uRBCs and rings (Fig. 4c). This fits with the reduced phosphorylation identified for S446 and S447 at trophozoites and schizonts stages. Paradoxically, although we observed increasing S729 and total B-Raf signals from rings to schizont stages on the array, this was not reflected in increased signal for full-length B-Raf on the western blotting. Instead, the increasing signal on the array corresponded to increasing bands on the western blotting that have lower molecular mass (see Supplementary Fig. 4 for additional data). Together, this indicates that B-Raf is indeed phosphorylated at the ring stage of $P$. falciparum development, before degradation in the later stages of parasite development (see "Discussion").

Overall, the microarray and western blotting data points to activation of B-Raf at ring stages and degradation at later stages. To assess whether parasite requirements for host B-Raf activation 
a

\begin{tabular}{|c|c|c|}
\hline Protein & $\begin{array}{l}\text { Phospho site } \\
\text { (human) }\end{array}$ & $\begin{array}{c}\text { Error } \\
(\%)\end{array}$ \\
\hline \multirow{3}{*}{ B-Raf } & S446 + S447 & 10 \\
\hline & S729 & 22 \\
\hline & Pan-specific & 25 \\
\hline \multirow{3}{*}{ C-Raf } & S296 & 10 \\
\hline & Pan-specific & 29 \\
\hline & Pan-specific & 46 \\
\hline \multirow{6}{*}{ MEK1 } & S298 & 11 \\
\hline & T292 & 3 \\
\hline & T386 & 4 \\
\hline & T386 & 10 \\
\hline & Pan-specific & 9 \\
\hline & Pan-specific & 23 \\
\hline MEK1/2 & $\mathrm{S} 218+\mathrm{S} 222$ & 17 \\
\hline \multirow{3}{*}{ MEK2 } & T394 & 11 \\
\hline & T394 & 10 \\
\hline & Pan-specific & 17 \\
\hline \multirow{6}{*}{ ERK1 } & T202 & 16 \\
\hline & T207 & 21 \\
\hline & Y204 & 10 \\
\hline & Y204 & 19 \\
\hline & Pan-specific & 20 \\
\hline & Pan-specific & 13 \\
\hline \multirow{2}{*}{ ERK2 } & $\mathrm{T} 185+\mathrm{Y} 187$ & 14 \\
\hline & Pan-specific & 10 \\
\hline
\end{tabular}

Trophozoites

\begin{tabular}{|c|c|}
\hline Fold change & $\begin{array}{l}\text { Error } \\
(\%)\end{array}$ \\
\hline 0.94 & 39 \\
\hline $2.53^{*}$ & 33 \\
\hline $2.36^{*}$ & 30 \\
\hline 1.25 & 7 \\
\hline 1.26 & 10 \\
\hline $2.92^{*}$ & 6 \\
\hline 0.84 & 7 \\
\hline 0.79 & 3 \\
\hline 0.82 & 9 \\
\hline 0.83 & 8 \\
\hline 0.81 & 11 \\
\hline $1.33^{*}$ & 5 \\
\hline 1.09 & 18 \\
\hline 0.70 & 5 \\
\hline 0.75 & 18 \\
\hline 0.83 & 10 \\
\hline 0.78 & 40 \\
\hline 1.25 & 34 \\
\hline 0.83 & 16 \\
\hline 2.91 & 19 \\
\hline 0.87 & 7 \\
\hline 1.07 & 14 \\
\hline $1.85^{\star}$ & 6 \\
\hline 1.25 & 9 \\
\hline
\end{tabular}

${ }^{*} p<0.05$

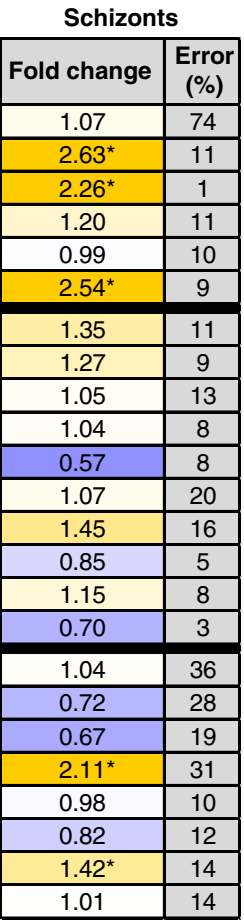

C

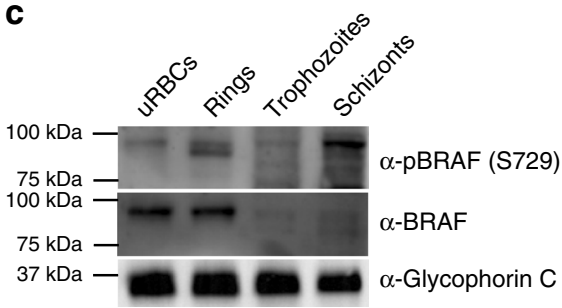

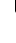

\begin{tabular}{|c|c|}
\hline Fold change & $\begin{array}{c}\text { Error } \\
(\%)\end{array}$ \\
\hline 1.07 & 74 \\
\hline $2.63^{*}$ & 11 \\
\hline $2.26^{*}$ & 1 \\
\hline 1.20 & 11 \\
\hline 0.99 & 10 \\
\hline $2.54^{*}$ & 9 \\
\hline 1.35 & 11 \\
\hline 1.27 & 9 \\
\hline 1.05 & 13 \\
\hline 1.04 & 8 \\
\hline 0.57 & 8 \\
\hline 1.07 & 20 \\
\hline 1.45 & 16 \\
\hline 0.85 & 5 \\
\hline 1.15 & 8 \\
\hline 0.70 & 3 \\
\hline 1.04 & 36 \\
\hline 0.72 & 28 \\
\hline 0.67 & 19 \\
\hline $2.11^{*}$ & 31 \\
\hline 0.98 & 10 \\
\hline 0.82 & 12 \\
\hline $1.42^{*}$ & 14 \\
\hline 1.01 & 14 \\
\hline
\end{tabular}

b

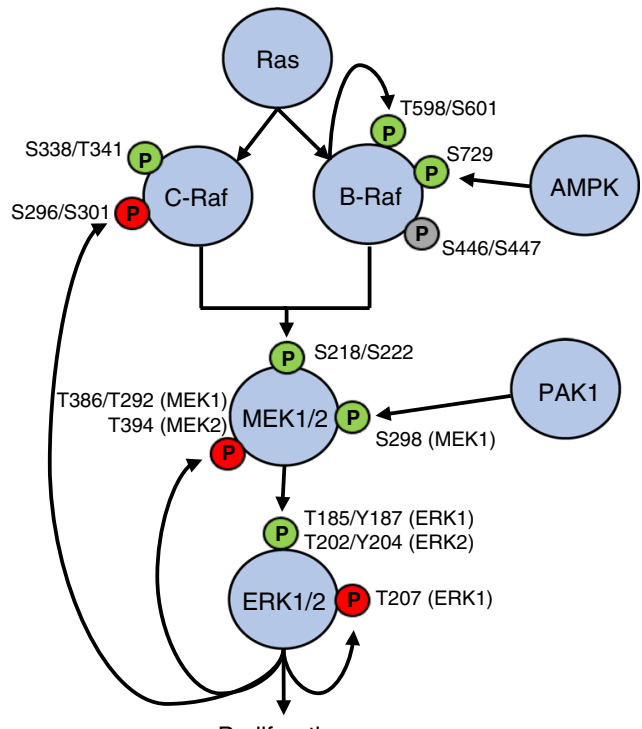

Proliferation
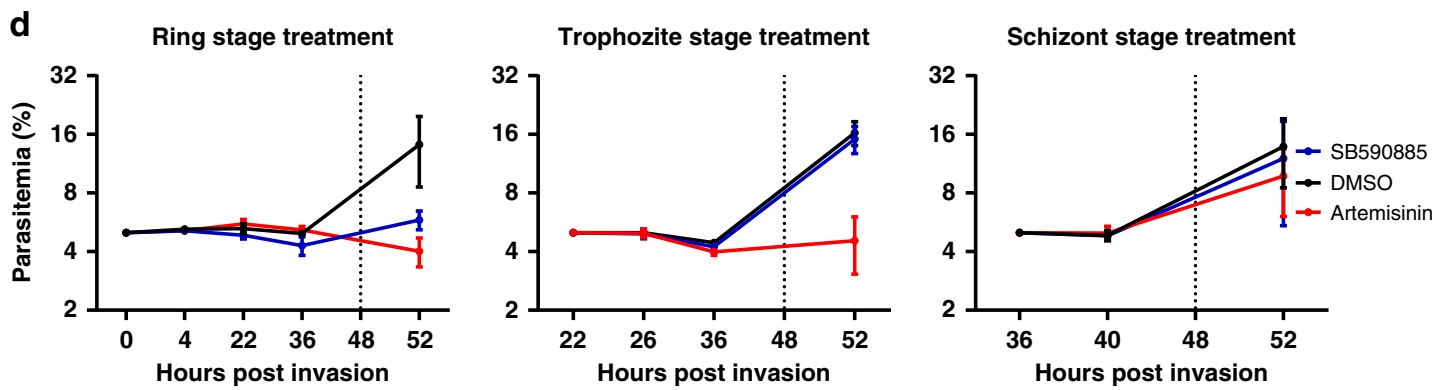

were also ring-stage-specific, as suggested by the apparent degradation of B-Raf in trophozoite- and schizont-infected cells (Fig. 4c), we treated highly synchronized parasites in $4 \mathrm{~h}$ windows with a potent B-Raf inhibitor (SB-590885, see below). Only when the treatment was applied at ring-stage did it display parasiticidal activity, whereas there was no effect when the cultures were treated at the trophozoite or schizont stage, in stark contrast to artemisinin, which displayed (as expected) a strong effect at the trophozoite stage (Fig. 4d).

Further supporting activation of Rafs in iRBCs, the B-Raf/CRaf substrates MEK1/2 showed increased phosphorylation on several residues at both the ring and schizont time points 
Fig. 4 Activation of a MAPK pathway in infected erythrocytes. a Summary table of the MAPK pathway components and phosphorylation sites covered by the antibody microarray, including RAF, MEK1/2 and ERK1/2 with the fold change from the uninfected erythrocyte control signal for each of the three time points (erythrocytes infected with rings, trophozoites or schizonts) shown. Signals that were significantly different during infection are marked by an asterisk ( $p<0.05$, two-tailed $T$-test). Phosphosites underlined in the table were flagged as cross-reactive with parasite material in Fig. 2 , but are included here for completeness. $\mathbf{b}$ Schematic representation of the MAPK pathway with the relevant phosphorylation sites present in the array data set covered in a. Green ' $P$ ' represents phosphorylation sites associated with the activity of the respective kinase, whereas the red ' $P$ 's represent negative feedback phosphorylation sites. The grey ' $P$ ' represents a known priming site on B-Raf. c Western blotting validation of B-Raf phosphorylation. A phospho-specific antibody to B-Raf (S729) detected phosphorylated B-Raf in ring-stage infected erythrocytes (upper panel). A pan-B-Raf antibody detected B-Raf in the uninfected and ring-stage-infected erythrocyte samples, with a strong decrease at the trophozoite and schizont stages (middle panel). An antibody against Glycophorin- $C$ was used as a loading control (lower panel), $n=3$ independent experiments. $\mathbf{d}$ Synchronized $P$. falciparum cultures were treated in $4 \mathrm{~h}$ windows with $2.45 \mu \mathrm{M}$ SB-590885 ( $5 \times \mathrm{IC}_{50}$, see Fig. 6), $0.091 \mu \mathrm{M}$ artemisinin or DMSO (vehicle control), starting at 0 (rings), 22 (trophozoites) and 38 (schizonts) h post invasion (the black arrows indicate the end of treatment, where the medium was replaced with fresh medium without drug). The parasitemia was then monitored until the next cycle of parasite development to assess parasite survival. Starting parasitemia was normalized to $5 \%$ across the biological replicates $(n=3)$ error bars represent the SEM. Source data are provided as a Source Data file.

(Fig. 4a). MEK1/2 phosphorylation of S298 (resulting from p21activated kinase [PAK] activity) during the late stages of $P$. falciparum infection has previously been established by western blotting analysis ${ }^{15}$, aligning with the 1.35-fold increase of pS298 at the schizont stage observed in the array experiment. Other phosphosites such as T292 and S218+ S222 (MEK1/2) show a signal increase in schizont-infected red cells. MEK1 T292 is involved in negative feedback of the kinase ${ }^{36}$, whereas S218+ S222 and S222 + S226 are the activation loop phosphosites in MEK1 and MEK2, respectively ${ }^{37,38}$. All the MEK1/2 phosphospecific antibodies, as well as the ERK1 (Y204) antibody, showed some cross-reactivity with parasite protein (underlined in Fig. 4a), which makes the overall MEK1/2 data difficult to interpret (Fig. 4a).

MEK1/2 phosphorylate ERK1 and ERK2 (on T202- 204 for ERK1, on T185 -Y187 for ERK2), and these residues are the only known targets of MEK1 activity 39,40 . ERK2 is slightly more phosphorylated in ring-infected cells than in uninfected erythrocytes (fold change $=1.09$ ); at the trophozoite stage, it has increased further (fold change $=1.85$ ), and at the schizont-stage ERK2 phosphorylation is still elevated (fold change $=1.42$ ). The array results for the ERK1 activation sites (T202 and Y204) at the ring-stage of $P$. falciparum development are however difficult to interpret, as these signals indicate increases and decreases as well. ERK1 T207 is a known autophosphorylation regulatory site and showed a fold change value of 1.25 in trophozoite-infected red cells-, supporting that regulation of ERK1 activity occurs during infection $^{41}$. These data support that $P$. falciparum iRBCs modify the host cell Raf/Mek/ERK pathway during growth, and that the activity of RBC B-Raf is required for parasite maturation beyond ring stages.

c-MET signalling in infected erythrocytes. Following the identification of the MAPK pathway activation, we focused our attention on signalling components that lay upstream of the MAPK pathway and found that the receptor tyrosine kinase cMET, which is known to signal to MAPK pathways in other systems ${ }^{42}$, was clearly activated by infection (Fig. 3). C-MET is expressed in many cells of mesenchymal origins ${ }^{43,44}$, and its activation occurs through the binding of hepatocyte growth factor (HGF) to the extracellular domain, prompting activation through homodimerization and trans-autophosphorylation ${ }^{45}$. CMET controls several key downstream pathways including (but not limited to) the MAPK pathway (proliferation, motility and cell cycle progression) and the phosphatidylinositol 3-kinase/ AKT pathway (cell survival) ${ }^{42}$. There is no homologue of c-MET (or any member of the tyrosine kinase group) in P. falciparum ${ }^{7}$.

The array contained a total of nine phospho-specific antibodies targeting c-MET, seven of which passed cross-reactivity and low- intensity signal thresholds (Fig. 5a). There was no noteworthy change in the phosphorylation of any of these residues at the ring stage. In contrast, five out of the seven sites show an increase in phosphorylation in trophozoite-infected cells (fold change $>1.38$, Fig. 5a). The signals from two antibodies against c-Met phosphorylation sites decreased at the trophozoite stage. One of these is directed against $\mathrm{Y} 1230+\mathrm{Y} 1234+\mathrm{Y} 1235$; this antibody is affected by high error between duplicates $(848 \%$ at the trophozoite stage) and was deemed unreliable. The other antibody recognizes pY1230 and is the only reliable c-MET antibody to show a decrease of phosphorylation at the trophozoite stage. At the schizont stage, most sites were slightly higher than the uninfected control. Two unique pan-specific cMET antibodies were also present on the array. Both pan-specific antibodies showed minimal signal variation between samples, indicating that the level of c-MET remained relatively constant as expected (Fig. 5a).

Antibodies against c-MET S975 and Y1349+ Y1356 were not present on the array or removed from analysis because of lowintensity signal, respectively. Reliable antibodies were available for pY1230 and pY1234 + pY1235, which lie within the c-MET catalytic domain and are phosphorylated in response to binding of HGF, activating the kinase 46 . Although increased phosphorylation was evident for Y1234 + Y1235 across life cycle stages, with a peak signal seen at trophozoites, this occurred only at schizont stage for Y1230. Increased Y1234 + Y1235 phosphorylation compared with uRBCs was confirmed by western blot analysis using independently sourced Y1234 + Y1235 and panMET antibodies (not used on the microarray), with peak signal again seen in trophozoites (Fig. 5c). This confirms that c-MET is indeed phosphorylated in the catalytic domain during trophozoite infection. As observed for B-Raf, we saw a decrease in apparent size of c-MET during the ring to trophozoite transition, which may be due to differences in phosphorylation status, or to partial degradation of the protein (see Discussion).

Phosphorylation of c-MET Y1003 peaks at the trophozoite stage. In nucleated cells, phosphorylated Y1003 recruits the cCBL (Casitas B-lineage lymphoma) protein, which promotes ubiquitination and degradation of c-MET via the $26 \mathrm{~S}$ proteasome $\mathrm{e}^{47,48}$. The activity of the $20 \mathrm{~S}$ proteasome has been previously reported in mature erythrocytes; in contrast, no $26 \mathrm{~S}$ activity was observed in these cells ${ }^{49}$. The absence of $26 \mathrm{~S}$ proteasomal activity in erythrocytes could explain why c-MET protein maintains a steady-state level throughout blood-stage parasite development (Fig. 5a), despite Y1003 phosphorylation.

Inhibitors of host kinases impair parasite proliferation. We previously published that U0126, a highly selective allosteric inhibitor of MEK1/2, as well as other structurally distinct selective 
a

\begin{tabular}{|c|c|}
\hline Phospho site (human) & $\begin{array}{c}\text { Error } \\
(\%)\end{array}$ \\
\hline Y1003 & 11 \\
\hline S1236 & 14 \\
\hline $\mathrm{T} 1241$ & 16 \\
\hline $\mathrm{Y} 1234+\mathrm{Y} 1235$ & 9 \\
\hline $\mathrm{Y} 1234+\mathrm{Y} 1235+\mathrm{S} 1236$ & 24 \\
\hline $\mathrm{Y} 1230+\mathrm{Y} 1234+\mathrm{Y} 1235$ & 26 \\
\hline $\mathrm{Y} 1230$ & 10 \\
\hline Pan-specific & 9 \\
\hline Pan-specific & 7 \\
\hline
\end{tabular}

\begin{tabular}{|c|c|}
\hline Fold change & $\begin{array}{c}\text { Error } \\
(\%)\end{array}$ \\
\hline 0.97 & 13 \\
\hline 1.13 & 6 \\
\hline 1.06 & 9 \\
\hline 1.28 & 15 \\
\hline 0.99 & 15 \\
\hline 0.77 & 954 \\
\hline 0.97 & 17 \\
\hline 0.88 & 16 \\
\hline 0.88 & 8 \\
\hline
\end{tabular}

\begin{tabular}{|c|c|}
\hline Fold change & $\begin{array}{c}\text { Error } \\
\text { (\%) }\end{array}$ \\
\hline 1.54 & 5 \\
\hline 1.38 & 1 \\
\hline 1.72 & 7 \\
\hline 1.52 & 6 \\
\hline 1.46 & 5 \\
\hline 0.74 & 848 \\
\hline 0.89 & 5 \\
\hline 0.66 & 3 \\
\hline 0.83 & 7 \\
\hline${ }^{*} \boldsymbol{p}<\mathbf{0 . 0 5}$ &
\end{tabular}

\begin{tabular}{|c|c|}
\hline Fold change & $\begin{array}{c}\text { Error } \\
(\%)\end{array}$ \\
\hline 1.19 & 8 \\
\hline 1.06 & 2 \\
\hline 1.19 & 8 \\
\hline 1.20 & 5 \\
\hline 0.83 & 18 \\
\hline 1.04 & 7 \\
\hline 1.24 & 15 \\
\hline 0.94 & 10 \\
\hline 0.87 & 16 \\
\hline
\end{tabular}

b

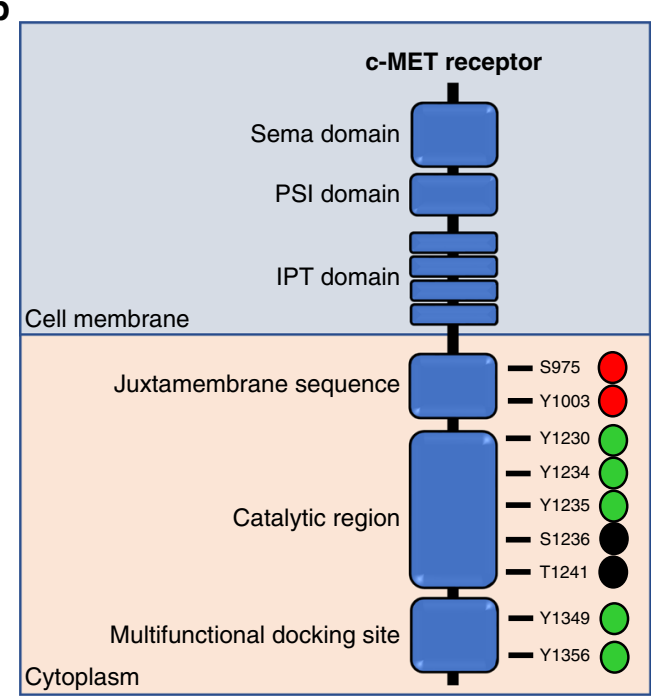

C

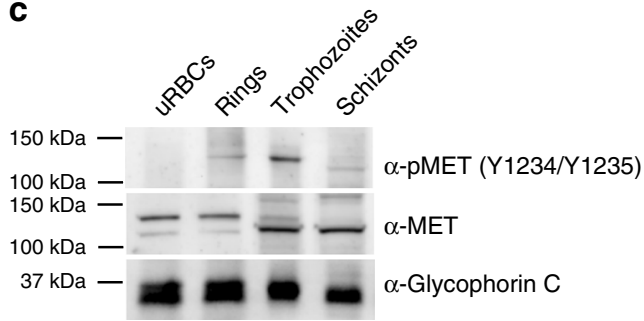

Fig. 5 Activation of c-MET in infected erythrocytes. a c-MET phosphorylation sites covered by the antibody microarray, with the fold change from uRBC control values for the three time points (ring, trophozoite and schizont). Signals that were significantly different during infection are marked by an asterisks $(p<0.05$, unpaired two-tailed T-test). b Schematic of the c-MET protein. The juxtamembrane sequence contains both S975 and Y1003, which represent cCBL-binding sites that target the receptor for degradation (red circles) ${ }^{73,74}$. The catalytic domain contains Y1230, Y1234, Y1235, S1236 and T1241. All activating tyrosine phosphorylations (green circles) occur through receptor homodimerization 42,45,75. The function of S1236 and T1241 (black circles) is uncharacterized. The multifunctional docking site contains two phosphosites, Y1349 and Y1356, which aid in the binding of adapter molecules such as grb2 and $\mathrm{GAB1}{ }^{45}$. c Western blotting validation of c-MET phosphorylation across asexual development. A phospho-specific antibody to c-MET (Y1234 + Y1235) detected phosphorylated c-MET at the ring and trophozoite stages (upper panel), $n=2$ independent experiments. A pan c-MET antibody detected c-MET across all four samples, with a double band in the uninfected, ring and trophozoite samples (middle panel). An antibody against Glycophorin-C was used as a loading control (lower panel). Source data are provided as a Source Data file.

inhibitors of these kinases, display low $\mu \mathrm{M}$ potency against $P$. falciparum in vitro ${ }^{15}$. The findings reported here that several additional human kinases such as c-MET and B-Raf are activated by infection raise the question of whether their inhibition would likewise impair parasite proliferation. Several selective inhibitors of c-MET and B-Raf have emerged from the cancer drug discovery pipeline, allowing us to measure the $\mathrm{IC}_{50}$ values in parasite proliferation assays of the following inhibitors: Crizotinib (an inhibitor of c-Met and the related receptor tyrosine kinase ALK [anaplastic lymphoma kinase]), PHA-665752 (a c-MET-selective inhibitor), SB-590885 (a B-Raf selective inhibitor) and Sorafenib (a B-Raf inhibitor, which also targets C-Raf, albeit with a tenfold lower potency $)^{50}$ (Fig. 6b, c). Despite the absence of B-Raf, cMET and ALK homologues in the Plasmodium kinome, all these inhibitors displayed low nano- to micromolar $\mathrm{IC}_{50}$ values against both P. falciparum and P. knowlesi in parasite proliferation assays (Fig. 6a). We cannot, at this stage, exclude off-target effects against Plasmodium proteins. However, our observation that the B-Raf inhibitor SB-590885 is effective only against ring stages, i.e., during the period where B-Raf is present and before its apparent degradation during the ring to trophozoite transition (Fig. 4a, d), together with the absence of c-MET or B-Raf homologues in the parasite's kinome, strongly suggests that inhibition of these host erythrocyte kinases impairs parasite proliferation. This in turn indicates that activation of these human kinases by malaria parasites may play an important role in their survival inside erythrocytes.

To determine at which point in the asexual cycle the parasite die if treated with c-MET inhibition at the start of the cycle, and to expand the aforementioned data pertaining to B-Raf inhibition (Fig. 4c), cultures of P. falciparum were highly synchronized to a $0-4 \mathrm{~h}$ post-invasion window of development. Cultures were treated with $5 \times \mathrm{IC}_{50}$ of SB-590885, Crizotinib, PHA-665752, the frontline antimalarial Artemisinin, or with the merozoite invasion inhibitor heparin. Parasites were monitored via flow cytometry every $12 \mathrm{~h}$ over $84 \mathrm{~h}$. The normalized live parasitemia detected for each time point was determined through co-staining with Hoechst 33342 (DNA stain) and Mito-tracker Orange 
a

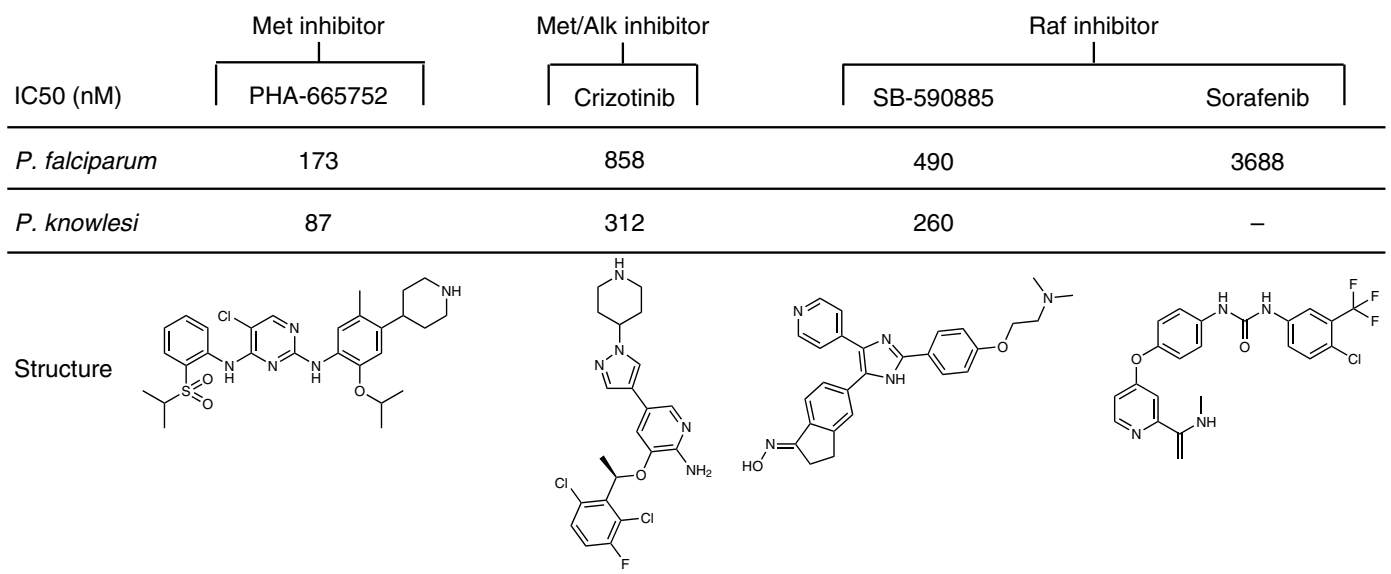

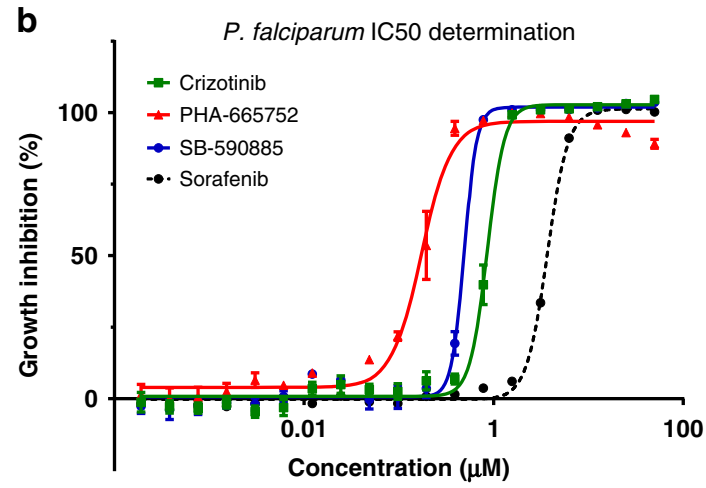

d

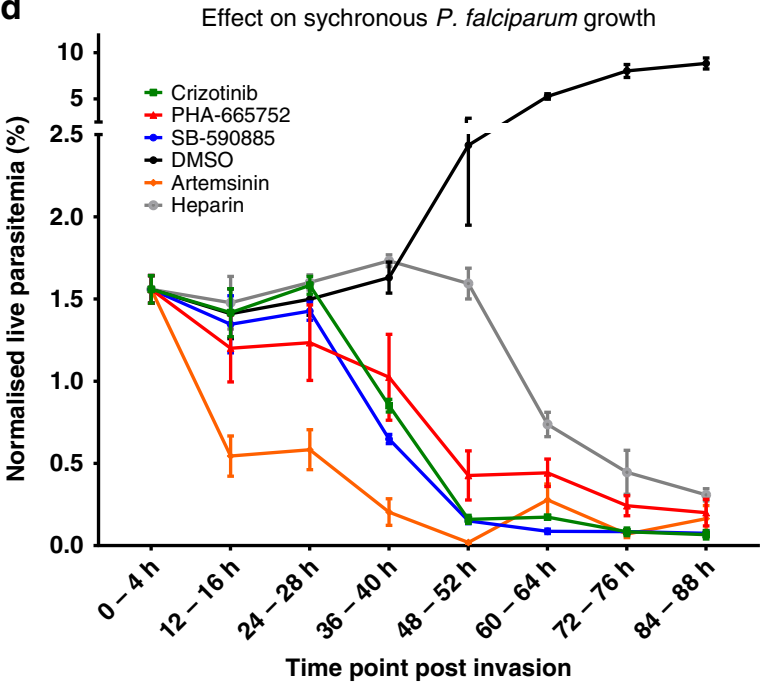

C

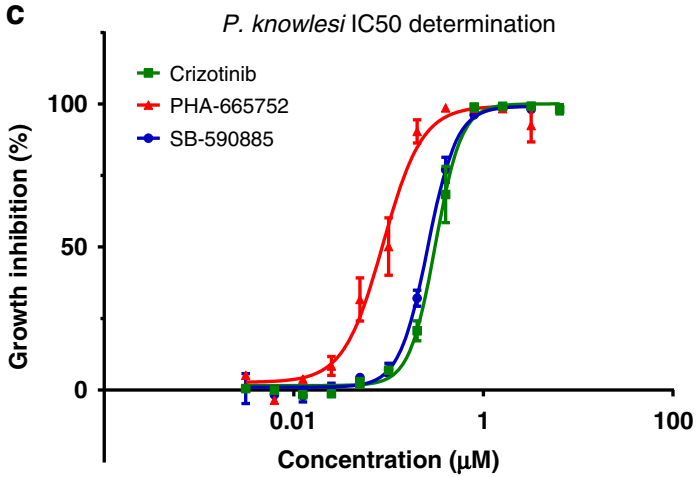

e

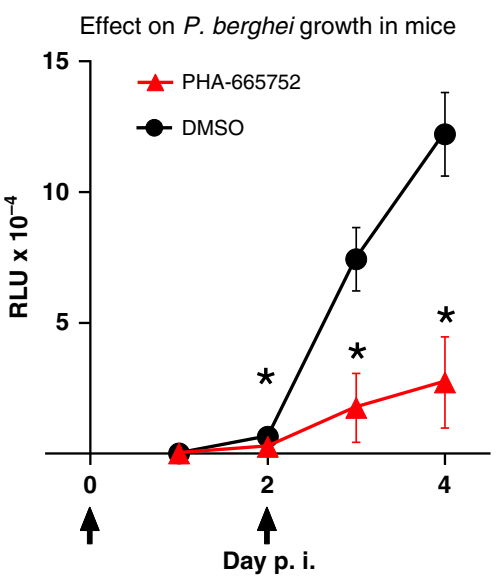

Fig. 6 IC $_{50}$ and phenotypic effect of human kinase inhibitors on Plasmodium blood-stage development. a IC 50 values of the human kinase inhibitors Crizotinib (MET/ALK inhibitor), PHA-665752 (Met inhibitor), SB-590885 (B-Raf inhibitor) and Sorafenib (B-Raf inhibitor, P. falciparum only) on asynchronous $P$. falciparum and ring-stage $P$. knowlesi asexual growth. $\mathbf{b}$ Growth inhibition curve for asynchronous $P$. falciparum, IC 50 measured after $72 \mathrm{~h}$ of compound exposure, $n=3, n=2$ for sorafenib ( $n$ represents biologically independent samples), data are presented as mean values \pm SEM. c Growth inhibition curve for $P$. knowlesi, $I C_{50}$ s measured after $50 \mathrm{~h}$ of compound exposure, $n=3$ biologically independent samples, data are presented as mean values \pm SEM. $\mathbf{d}$ Effect of treatment with $5 \times$ the $\mathrm{IC}_{50}$ value of the compounds Crizotinib, PHA-665752, SB-590885, Artemisinin, DMSO (equivalent volume of DMSO in Crizotinib sample) and heparin $(15 \mu \mathrm{l} / \mathrm{ml}$ ) on highly synchronous ( $4 \mathrm{~h}$ window) P. falciparum cultures over $88 \mathrm{~h}$ ( $n=5 \mathrm{biologically} \mathrm{independent}$ samples). The $Y$-axis represents the live parasitemia values for each time point normalized to a starting total parasitemia of $2 \%$, data are presented as mean values \pm SEM. e Effect of treating mice with PHA-665752 on the growth of $P$. berghei. The graph shows relative luminescence units (RLU) in the blood of BALB/c mice infected with parasites constitutively expressing firefly luciferase and treated or not with PHA- 665752 at $25 \mathrm{mg} / \mathrm{kg}$ at times indicated by arrows. ${ }^{\star} p<0.01$ by two-tailed $T$-test; $n=7$ mice per treatment group, data are presented as mean values \pm SEM. Source data are provided as a Source Data file. 
(mitochondrial potential stain). The average values of the biological replicates are represented graphically in Fig. 6d. Treatment with Artemisinin resulted in a reduction in live parasitemia at $12-16 \mathrm{hpi}$, indicative of ring-stage parasite death. Treatment with heparin caused a drop in live parasitemia post 48 hpi, as expected for an invasion inhibitor. In contrast, the first notable reduction in parasitemia observed after treatment with Crizotinib, PHA-665752 or SB-590885 occurs prior to the schizont stage. This indicates that all three human kinase inhibitors exert their largest effect on parasite survival at a time that is consistent with the activation time of their target kinases (and, in the case of B-Raf, with the time where the full-length protein is present, i.e., ring stage), as revealed by the microarray and Western blot analysis. Notably, all compounds except heparin resulted in a live parasitemia below $0.2 \%$ by $84-88 \mathrm{hpi}$, with SB-590885 and Crizotinib reducing live parasitemia to $<0.1 \%$. For an initial assessment of the in vivo efficacy of the cMET inhibitor PHA-665752, we treated mice infected with the rodent malaria parasite $P$. berghei. A treatment regime and formulation that was previously used to control tumour growth in mice with the same inhibitor ${ }^{51}$ led to a 3.3 -fold reduction in parasite load after two doses of treatment in vivo (Fig. 6e, day 4).

\section{Discussion}

The antibody microarray analysis generated a large number of testable hypotheses on the involvement of host erythrocyte signalling proteins during infection by the human malaria parasite P. falciparum. We achieved western blotting validation for three of the host kinases (MEK1 [15], c-Met [this study] and B-Raf [this study]), which the array data suggest are activated by infection. In the other infection systems that we characterized through this approach (hepatocyte/hepatitis C virus ${ }^{52}$ and mosquito cells/Wolbachia endosymbiont ${ }^{53}$ ), the functional relevance of the most interesting array hits was established through small interfering RNA (siRNA) campaigns. In the erythrocyte/Plasmodium system where siRNA-based knockdown of host genes is not feasible, we propose that similar western blotting validation should be undertaken as the first step in further work on any of the numerous additional enzymes/pathways identified as hits in the array.

The need for systematic validation notwithstanding, the extent to which host erythrocyte signalling is modulated by Plasmodium infection is surprising. The array data are consistent with earlier observations implicating some of the host cell signalling pathways in the infection process, and allow an in-depth, highly specific understanding of the mobilization of such pathways (e.g., see above the section pertaining to PKC isoforms). The observation (Fig. 4c) that B-Raf is present at the ring-stage but appears to be degraded in trophozoite- and schizont-infected RBCs is consistent with a thorough modulation of host erythrocyte signalling pathways during infection, not only through activation/inactivation of enzymes by phosphorylation, but also through affecting the abundance of specific signalling elements. In line with this observation, the host erythrocyte cAMP-dependent kinase PKA has been reported to be similarly degraded in infected cells ${ }^{54}$. This is reminiscent of the strategy of other intracellular pathogens to modify the composition of signalling pathways in their host cell. A well-characterized example is that of the Lethal Factor, a component of the anthrax toxin that is secreted by Bacillus anthracis, and that possesses highly selective endopeptidase activity directed against host cell MEKs, thereby inactivating MAPK pathways (reviewed in ref. ${ }^{55}$ ). Downregulation of MAPK pathways through degradation of specific components is not restricted to host-pathogen interactions. For example, during hematopoietic cell apoptosis, IL-3-deprivation results in the cleavage of Raf-1 by caspase 9, causing separation of the $\mathrm{N}$ terminal regulatory domain from the C-terminal kinase domain $^{56}$. Likewise, c-MET and several receptor tyrosine kinases $^{57}$ can undergo specific proteolytic cleavage events, which mediate various biological outcomes (reviewed in ref. ${ }^{58}$ ). It is therefore interesting to note that in our western blotting analysis, c-MET appears as a band of lower mass in trophozoite-/schizontinfected RBCs than at the ring stage (Fig. 5). It is tempting to speculate that intracellular pathogens hijack such cellular processes to suppress host pathways. Our observation that host erythrocyte B-Raf is activated early in infection (ring-stage), and apparently subsequently degraded, warrants further studies that will yield fascinating insights into host-parasite interactions. In particular, it will be of great interest to investigate if some host pathways identified as activated in the present study are dependent on the replacement of specific host cell kinases by parasiteencoded kinases, several of which are known to be exported to the host erythrocyte (e.g., see refs. ${ }^{59,60}$.).

A major long-term objective of this research is the identification of antimalarial compounds with untapped modes of action. It is a clear possibility that part of the erythrocyte signalling response to infection may be directed towards activating innate immune defence mechanisms, and thus downregulating such responses would be undesirable. However, based on the hypothesis that at least some of the signalling events triggered in the host cell by infection are required for parasite survival, we tested highly selective inhibitors against some of the activated kinases and showed that these compounds have high potency (in the low $\mathrm{nM}$ range) in in vitro culture systems. The nanomolar activity against both $P$. falciparum and the phylogenetically distant $P$. knowlesi for c-Met and B-Raf inhibitors indicate that the reliance on the activation of host erythrocyte pathways spans various Plasmodium species. Importantly, the markedly impaired proliferation of $P$. berghei in a murine malaria model treated with the c-Met inhibitor PHA-665752 shows that targeting host kinases to control malaria infection in vivo is achievable, strengthening the case for kinase-focussed host-targeted intervention.

Major attractive features of targeting host cell kinases as a strategy to develop novel antimalarials are threefold: first is the effectiveness of specific inhibitors across phylogenetically distant Plasmodium species, illustrated by the facts (i) that inhibitors against three host cell kinases activated by infection (MEK1, cMet and B-Raf) display similar in vitro potency on $P$. falciparum and $P$. knowlesi, and (ii) that MEK and c-Met inhibitors also have activity against the $P$. berghei rodent malaria parasite ex vivo $\left(\mathrm{MEK}^{15}\right)$ and in vivo (c-MET). A second tremendous advantage of this strategy is that the most parsimonious pathway to resistance, i.e., the selection under drug pressure of genotypes encoding a mutated target with decreased susceptibility, is not possible if the target is encoded by the host. Third, large kinasedirected compound libraries are available from the intensive efforts made over the past two decades in the context of cancer chemotherapy (including inhibitors of many of the kinases identified in this study as being activated in infected erythrocytes), providing a unique repository of potential antimalarials. These can now be exploited as a basis for fundamental research into host-parasite interactions, and as leads for the development of novel antimalarials with untapped modes of action.

\section{Methods}

Plasmodium spp. culture and life-stage synchronization. Human erythrocytes were supplied by the Australian Red Cross. P. falciparum (clone 3D7) and $P$. knowlesi (YH1) were grown in human erythrocytes as previously described ${ }^{61-64}$. $P$. falciparum synchronization ( $4 \mathrm{~h}$ window) was achieved using $5 \% \mathrm{w} / \mathrm{v}$ Sorbitol and 
$15 \mu \mathrm{l} / \mathrm{ml}$ culture of heparin ${ }^{65}$. Both techniques were utilized for the trophozoiteand schizont-stage samples used on the antibody microarray, whereas an $8 \mathrm{~h}$ synchronization window was achieved through sorbitol alone for the ring stage. Purification of $P$. falciparum trophozoite and schizont-infected cells was achieved using a super-MACS column ${ }^{66}$. P. knowlesi cultures for in vitro assays were partially synchronized $\left(\sim 12 \mathrm{~h}\right.$ age range) using heparin ${ }^{62}$.

Kinexus antibody microarray preparation. The Kinex 900P array kits were purchased from Kinexus. Protein extracts were prepared as per the manufacturer's instruction. Each array was, unless otherwise specified, loaded with protein extracts normalized to equivalent cell number using a hemocytometer. Array scanning was conducted by the manufacturer at their facility. Arrays were loaded with protein extracts of rings $4-12 \mathrm{~h}$ ( $\sim 33 \%$ parasitemia, $n=3$ ), trophozoites $24-28 \mathrm{~h} \mathrm{(mag-}$ net purified, $n=3$ ) and schizonts $44-48 \mathrm{~h}$ (magnet purified, $n=2$ ). Parallel uRBC control extracts containing RBCs from the same donor as the iRBC treatment were loaded onto the arrays to measure baseline RBC phosphorylation (these cells were cultured in the same conditions as the parasite-infected samples for $\sim 24 \mathrm{~h}$ before collection). To identify phosphosites of parasite proteins cross-reactive to the array, saponin pellets and supernatants of magnet purified, saponin-lysed trophozoites/ schizonts were compared. Saponin pellet samples were loaded at $2 \mathrm{mg} / \mathrm{ml}$ on the array, $20 \times$ more pellet material than is present in $2 \mathrm{mg} / \mathrm{ml}$ of equivalent saponin supernatant material.

Western blotting. Western blotting analysis of infected and uninfected erythrocytic material performed on protein extracts prepared by resuspending cells in M-PER mammalian protein extraction reagent (Pierce) supplemented with $1 \times$ protease inhibitory cocktail (EDTA free) (Roche), $300 \mathrm{mM}$ benzamidine, $200 \mathrm{mM}$ phenylmethylsulfonyl fluoride, $500 \mathrm{mM}$ sodium fluoride, $100 \mathrm{mM}$ sodium orthovanadate and $500 \mathrm{mM} \beta$-glycerophosphate. Lysates were cleared by centrifugation $\left(10,000 \times g\right.$ for $15 \mathrm{~min}$ at $\left.4^{\circ} \mathrm{C}\right)$; samples were heated $\left(70^{\circ} \mathrm{C}\right)$ in Laemmli reducing sample buffer before separation on $4-12 \%$ Tris/Bis gradient SDS-polyacrylamide gel electrophoresis gels (Invitrogen). After electrophoresis, proteins were transferred to nitrocellulose membrane (Amersham) and blocked ( $1 \mathrm{~h}$ at room temperature) with 5\% Skim milk (Diploma) in Tris-buffered saline containing $0.05 \%$ Tween 20. Immunoblotting was performed using the following antibodies: phosphorylated c-MET (Y1234/Y1235; catalogue number 3077S, Cell Signaling Technologies), pan c-MET (catalogue number 8198, Cell Signaling Technologies), panB-Raf (catalogue number OTI4B2, Bio-Rad), phosphorylated B-Raf (S729, catalogue number AB-PK535, Kinexus) and Glycophorin-C (catalogue number ab175257, Abcam). All primary antibodies used at 1:500 dilution and incubated overnight at $4{ }^{\circ} \mathrm{C}$. Anti-Rabbit/Mouse-horseradish peroxidase-conjugated secondary antibody was used for all experiments at $1: 2500$ dilution (Monoclonal antibody facility Monash University Clayton, VIC, Australia). Full western blottings are available in Supplementary Fig. 6.

IC $_{50}$ determination. P. falciparum-The $\mathrm{IC}_{50}$ values of Crizotinib, PHA-665752, SB-590885, Sorafenib and Artemisinin were determined with asynchronous cultures with a starting parasitemia of $0.25 \%$ in $2 \%$ haematocrit. Cultures were incubated with the compounds for $72 \mathrm{~h}$ before adding SYBR-gold nucleic acid stain ( $1: 10,000$ dilution) (ThermoFisher Scientific) modified from ref. ${ }^{67}$ substituting SYBR-green for SYBR-gold. Plates were incubated with SYBR-gold for $1 \mathrm{~h}$ in the dark prior to reading fluorescence on a Tecan plate reader (I-control software) and the inhibitory $\mathrm{IC}_{50}$ determined using GraphPad PRISM (GraphPad software).

$P$. knowlesi-P. knowlesi growth inhibition assays using ring-stage parasites were set up at $1 \%$ parasitaemia and $1 \%$ haematocrit in 96 -well round-bottom plates at a final volume of $45 \mu \mathrm{L}$ as described ${ }^{68,69}$. A $10 \times$ final concentration of drug and controls was added to make the final volume to $50 \mu \mathrm{L}$ and the drug assay cultured for $50 \mathrm{~h}$ until parasites reached late trophozoites in the next growth cycle. Assays were stained with $10 \mu \mathrm{g} / \mathrm{mL}$ ethidium bromide (EtBr, Bio-Rad) for $1 \mathrm{~h}$ and washed prior to flow cytometry (Becton Dickinson LSR) assessment of parasitaemia with gating as per established protocols ${ }^{70}$. Parasitaemia counts were quantitated using FlowJo software (Tree Star) and the inhibitory $\mathrm{IC}_{50}$ determined using GraphPad PRISM (GraphPad Software).

Time-dependent inhibitor treatment of parasites. Highly synchronous cultures $(0-4 \mathrm{~h}$ post invasion) at $2 \%$ haematocrit treated with dimethyl sulfoxide (DMSO) (vehicle), Crizotinib, PHA-665752, SB-590885 or artemisinin at $5 \times$ the IC $_{50}$ values were monitored at $12 \mathrm{~h}$ time intervals over two full asexual intra-erythrocytic cycles $(84 \mathrm{~h})$ by flow cytometry. Live parasitaemia was quantitated by a dual-colour flow cytometry staining protocol using $2 \mu \mathrm{M}$ Hoechst 33342 staining for $8 \mathrm{~min}$ and $75 \mathrm{nM}$ MitoTracker Orange for $25 \mathrm{~min}$. Staining was completed in v-bottom plates with two washes in complete RPMI. Stained cells were transferred to polypropylene tubes and diluted one in four in complete RPMI. Cells were then immediately analysed on an LSR BDFortessa ${ }^{\mathrm{TM}}$ with the laser UV379 and filter 450/50 for Hoechst-33242 staining, and laser YG585 with filter 585/15 for MitoTracker Orange staining. The gating strategy used is available in Supplementary Fig. 5. Biological replicates $(n=5)$ were normalized to a $2 \%$ total parasitemia value at the first time point $(0-4 \mathrm{~h}$ post invasion).
P. falciparum stage-dependent inhibitor washout assay. P. falciparum cultures were synchronized into a $6 \mathrm{~h}$ development window at $2 \%$ haematocrit and treated with $5 \times$ the $72 \mathrm{~h}$ IC50 of SB-590885, Artemisinin or DMSO at 0-6 hpi (ring-stage treatment), 22-28 hpi (trophozoite-stage treatment) and 38-44 hpi (schizont-stage treatment). Treatments were washed from the respective cultures after $4 \mathrm{~h}$ (denoted by the black arrow) and monitored until the next cycle of parasite development to assess parasite survival using flow cytometry $(2 \mu \mathrm{M}$ Hoechst 33342 staining for 8 min). Starting parasitemia was normalized to $5 \%$ across the biological replicates $(n=3)$. Error bars represent the SEM.

P. berghei in vivo drug treatment assay. Animal research was conducted under licences from the UK Home Office and protocols were approved by the Animal Welfare and Ethical Review Body of the Wellcome Sanger Institute. Mice were purchased from Envigo, kept in specific-pathogen-free conditions and subjected to regular pathogen monitoring by sentinel screening. They were housed in individually ventilated cages furnished with autoclaved aspen woodchip, fun tunnel and Nestlets at $21^{\circ} \mathrm{C} \pm 2{ }^{\circ} \mathrm{C}$ under a $12: 12 \mathrm{~h}$ light-dark cycle at a relative humidity of $55 \pm 10 \%$. They were fed a commercially prepared autoclaved dry rodent diet and water, both available ad libitum. The health of animals was monitored by routine daily visual health checks. Cohorts of seven female, 10 -week-old BALB/c mice per treatment group were infected by intravenous inoculation with $10^{6}$ infected erythrocytes. Assays to determine parasite growth were performed as described ${ }^{71}$, using the reporter parasite line PbGFPLuc $c_{\text {con }}$ (RMgm-29 in the rodent malaria genetic modification database, http://www.pberghei.eu/index.php?.rmgm=29), which expresses a green fluorescent protein-firefly luciferase fusion protein under the control of the constitutive eef1a promoter $^{72}$. Treatment with PHA-665752 or solvent control was $3 \mathrm{~h}$ thereafter and on day two post infection by intraperitoneal injection of 25 $\mathrm{mg} / \mathrm{kg} / \mathrm{day}$ of PHA- 665752 . The drug was prepared fresh by diluting a stock of $50 \mathrm{mg}$ PHA-665752 in $0.5 \mathrm{ml}$ DMSO (w/v) in water $(1: 40, \mathrm{v} / \mathrm{v})$. The same drug formulation and treatment regime had proved effective in controlling carcinoma growth ${ }^{51}$.

Reporting summary. Further information on research design is available in the Nature Research Reporting Summary linked to this article.

\section{Data availability}

Raw data pertaining to this study are available in Supplementary Data 1-3. Source data are provided with this paper.

Received: 3 June 2020; Accepted: 16 July 2020; Published online: 11 August 2020

\section{References}

1. Singh, B. et al. A large focus of naturally acquired Plasmodium knowlesi infections in human beings. Lancet 363, 1017-1024 (2004).

2. Brasil, P. et al. Outbreak of human malaria caused by Plasmodium simium in the Atlantic Forest in Rio de Janeiro: a molecular epidemiological investigation. Lancet Glob. Health 5, e1038-e1046 (2017).

3. WHO. World Malaria Report (WHO, 2017).

4. Haldar, K., Bhattacharjee, S. \& Safeukui, I. Drug resistance in Plasmodium. Nat. Rev. Microbiol. 16, 156-170 (2018).

5. Bhullar, K. S. et al. Kinase-targeted cancer therapies: progress, challenges and future directions. Mol. Cancer 17, 48 (2018).

6. Roskoski, R. Jr. Properties of FDA-approved small molecule protein kinase inhibitors. Pharm. Res. 144, 19-50 (2019).

7. Ward, P., Equinet, L., Packer, J. \& Doerig, C. Protein kinases of the human malaria parasite Plasmodium falciparum: the kinome of a divergent eukaryote. BMC Genomics 5, 79 (2004).

8. Lucet, I. S., Tobin, A., Drewry, D., Wilks, A. F. \& Doerig, C. Plasmodium kinases as targets for new-generation antimalarials. Future Med Chem. 4, 2295-2310 (2013).

9. Schor, S. \& Einav, S. Combating intracellular pathogens with repurposed hosttargeted drugs. ACS Infect. Dis. 4, 88-92 (2018).

10. Deitsch, K. W. Self-targeting blocks malaria parasite invasion. J. Exp. Med. 212, 1143 (2015).

11. Zenonos, Z. A. et al. Basigin is a druggable target for host-oriented antimalarial interventions. J. Exp. Med. 212, 1145-1151 (2015).

12. Murphy, S. C. et al. Erythrocyte G protein as a novel target for malarial chemotherapy. PLoS Med. 3, e528 (2006).

13. Harrison, T. et al. Erythrocyte G protein-coupled receptor signaling in malarial infection. Science 301, 1734-1736 (2003).

14. Ntumngia, F. B., Thomson-Luque, R., Pires, C. V. \& Adams, J. H. The role of the human Duffy antigen receptor for chemokines in malaria susceptibility: 
current opinions and future treatment prospects. J. Receptor Ligand Channel Res. 9, 1-11 (2016).

15. Sicard, A. et al. Activation of a PAK-MEK signalling pathway in malaria parasite-infected erythrocytes. Cell Microbiol. 13, 836-845 (2011).

16. Hall, B. S., Daramola, O. O., Barden, G. \& Targett, G. A. Modulation of protein kinase $\mathrm{C}$ activity in Plasmodium falciparum-infected erythrocytes. Blood 89, 1770-1778 (1997).

17. Brizuela, M. et al. Treatment of erythrocytes with the 2-cys peroxiredoxin inhibitor, Conoidin A, prevents the growth of Plasmodium falciparum and enhances parasite sensitivity to chloroquine. PLoS ONE 9, e92411 (2014).

18. Koncarevic, S. et al. The malarial parasite Plasmodium falciparum imports the human protein peroxiredoxin 2 for peroxide detoxification. Proc. Natl Acad. Sci. USA 106, 13323-13328 (2009).

19. Carvalho, T. G. et al. The ins and outs of phosphosignalling in Plasmodium: parasite regulation and host cell manipulation. Mol. Biochem Parasitol. 208, 2-15 (2016).

20. Park, E. R., Eblen, S. T. \& Catling, A. D. MEK1 activation by PAK: a novel mechanism. Cell Signal. 19, 1488-1496 (2007).

21. Radu, M., Semenova, G., Kosoff, R. \& Chernoff, J. PAK signalling during the development and progression of cancer. Nat. Rev. Cancer 14, 13-25 (2014).

22. Elandalloussi, L. M. \& Smith, P. J. Preparation of pure and intact Plasmodium falciparum plasma membrane vesicles and partial characterisation of the plasma membrane ATPase. Malar. J. 1, 6 (2002).

23. Millholland, M. G. et al. A host GPCR signaling network required for the cytolysis of infected cells facilitates release of apicomplexan parasites. Cell Host Microbe 13, 15-28 (2013).

24. Bouyer, G. et al. Plasmodium falciparum infection induces dynamic changes in the erythrocyte phospho-proteome. Blood Cells Mol. Dis. 58, 35-44 (2016).

25. Welman, A., Griffiths, J. R., Whetton, A. D. \& Dive, C. Protein kinase C delta is phosphorylated on five novel Ser/Thr sites following inducible overexpression in human colorectal cancer cells. Protein Sci. 16, 2711-2715 (2007).

26. Liu, Y., Graham, C., Li, A., Fisher, R. J. \& Shaw, S. Phosphorylation of the protein kinase C-theta activation loop and hydrophobic motif regulates its kinase activity, but only activation loop phosphorylation is critical to in vivo nuclear-factor-kappaB induction. Biochem J. 361, 255-265 (2002).

27. Wang, X., Chuang, H. C., Li, J. P. \& Tan, T. H. Regulation of PKC-theta function by phosphorylation in T cell receptor signaling. Front Immunol. 3, 197 (2012)

28. Zhang, B. H. \& Guan, K. L. Activation of B-Raf kinase requires phosphorylation of the conserved residues Thr598 and Ser601. EMBO J. 19, 5429-5439 (2000).

29. Chong, H., Lee, J. \& Guan, K. L. Positive and negative regulation of Raf kinase activity and function by phosphorylation. EMBO J. 20, 3716-3727 (2001).

30. Leicht, D. T. et al. Raf kinases: function, regulation and role in human cancer. Biochim. Biophys. Acta 1773, 1196-1212 (2007).

31. Dorin-Semblat, D. et al. Functional characterization of both MAP kinases of the human malaria parasite Plasmodium falciparum by reverse genetics. Mol. Microbiol. 65, 1170-1180 (2007).

32. Lake, D., Correa, S. A. \& Muller, J. Negative feedback regulation of the ERK1/2 MAPK pathway. Cell Mol. Life Sci. 73, 4397-4413 (2016).

33. Brummer, T. et al. Functional analysis of the regulatory requirements of B-Raf and the B-Raf(V600E) oncoprotein. Oncogene 25, 6262-6276 (2006).

34. Mason, C. S. et al. Serine and tyrosine phosphorylations cooperate in Raf-1, but not B-Raf activation. EMBO J. 18, 2137-2148 (1999).

35. Brummer, T., Shaw, P. E., Reth, M. \& Misawa, Y. Inducible gene deletion reveals different roles for B-Raf and Raf-1 in B-cell antigen receptor signalling. EMBO J. 21, 5611-5622 (2002).

36. Eblen, S. T. et al. Mitogen-activated protein kinase feedback phosphorylation regulates MEK1 complex formation and activation during cellular adhesion. Mol. Cell Biol. 24, 2308-2317 (2004).

37. Roskoski, R. Jr. MEK1/2 dual-specificity protein kinases: structure and regulation. Biochem. Biophys. Res. Commun. 417, 5-10 (2012).

38. Bromberg-White, J. L., Andersen, N. J. \& Duesbery, N. S. MEK genomics in development and disease. Brief. Funct. Genomics 11, 300-310 (2012).

39. Payne, D. M. et al. Identification of the regulatory phosphorylation sites in pp42/mitogen-activated protein kinase (MAP kinase). EMBO J. 10, 885-892 (1991).

40. Goetz, E. M., Ghandi, M., Treacy, D. J., Wagle, N. \& Garraway, L. A. ERK mutations confer resistance to mitogen-activated protein kinase pathway inhibitors. Cancer Res. 74, 7079-7089 (2014).

41. Lai, S. \& Pelech, S. Regulatory roles of conserved phosphorylation sites in the activation T-loop of the MAP kinase ERK1. Mol. Biol. Cell 27, 1040-1050 (2016).

42. Organ, S. L. \& Tsao, M. S. An overview of the c-MET signaling pathway. Ther. Adv. Med. Oncol. 3, S7-S19 (2011)
43. Zarnegar, R. \& Michalopoulos, G. K. The many faces of hepatocyte growth factor: from hepatopoiesis to hematopoiesis. J. Cell Biol. 129, 1177-1180 (1995)

44. Teofili, L. et al. Expression of the c-met proto-oncogene and its ligand, hepatocyte growth factor, in Hodgkin disease. Blood 97, 1063-1069 (2001).

45. Rodrigues, G. A. \& Park, M. Autophosphorylation modulates the kinase activity and oncogenic potential of the Met receptor tyrosine kinase. Oncogene 9, 2019-2027 (1994).

46. Emaduddin, M., Bicknell, D. C., Bodmer, W. F. \& Feller, S. M. Cell growth, global phosphotyrosine elevation, and c-Met phosphorylation through Src family kinases in colorectal cancer cells. Proc. Natl Acad. Sci. USA 105, 2358-2362 (2008)

47. Peschard, P. et al. Mutation of the c-Cbl TKB domain binding site on the Met receptor tyrosine kinase converts it into a transforming protein. Mol. Cell 8 , 995-1004 (2001).

48. Jeffers, M., Taylor, G. A., Weidner, K. M., Omura, S. \& Vande Woude, G. F. Degradation of the Met tyrosine kinase receptor by the ubiquitin-proteasome pathway. Mol. Cell Biol. 17, 799-808 (1997).

49. Neelam, S., Kakhniashvili, D. G., Wilkens, S., Levene, S. D. \& Goodman, S. R. Functional $20 \mathrm{~S}$ proteasomes in mature human red blood cells. Exp. Biol. Med. (Maywood) 236, 580-591 (2011).

50. King, A. J. et al. Demonstration of a genetic therapeutic index for tumors expressing oncogenic BRAF by the kinase inhibitor SB-590885. Cancer Res. 66, 11100-11105 (2006)

51. Zhi, J. et al. Effects of PHA-665752 and vemurafenib combination treatment on in vitro and murine xenograft growth of human colorectal cancer cells with BRAF(V600E) mutations. Oncol. Lett. 15, 3904-3910 (2018).

52. Haqshenas, G. et al. Signalome-wide assessment of host cell response to hepatitis C virus. Nat. Commun. 8, 15158 (2017).

53. Haqshenas, G. et al. A role for the insulin receptor in the suppression of dengue virus and Zika virus in Wolbachia-infected mosquito cells. Cell Rep. 26, 529-535 e523 (2019).

54. Syin, C. et al. The H89 cAMP-dependent protein kinase inhibitor blocks Plasmodium falciparum development in infected erythrocytes. Eur. J. Biochem. 268, 4842-4849 (2001).

55. Turk, B. E. Exceptionally selective substrate targeting by the metalloprotease anthrax lethal factor. Adv. Exp. Med. Biol. 1111, 189-203 (2019).

56. Cornelis, S. et al. Apoptosis of hematopoietic cells induced by growth factor withdrawal is associated with caspase- 9 mediated cleavage of Raf-1. Oncogene 24, 1552-1562 (2005).

57. Merilahti, J. A. M. \& Elenius, K. Gamma-secretase-dependent signaling of receptor tyrosine kinases. Oncogene 38, 151-163 (2019).

58. Fernandes, M., Duplaquet, L. \& Tulasne, D. Proteolytic cleavages of MET: the divide-and-conquer strategy of a receptor tyrosine kinase. BMB Rep. 52, 239-249 (2019).

59. Dorin-Semblat, D. et al. Malaria parasite-infected erythrocytes secrete PfCK1, the Plasmodium homologue of the pleiotropic protein kinase casein kinase 1. PLOS ONE 10, e0139591 (2015).

60. Nunes, M. C., Goldring, J. P., Doerig, C. \& Scherf, A. A novel protein kinase family in Plasmodium falciparum is differentially transcribed and secreted to various cellular compartments of the host cell. Mol. Microbiol. 63, 391-403 (2007).

61. Trager, W. \& Jensen, J. B. Human malaria parasites in continuous culture. Science 193, 673-675 (1976)

62. Lyth, O. et al. Cellular dissection of malaria parasite invasion of human erythrocytes using viable Plasmodium knowlesi merozoites. Sci. Rep. 8, 10165 (2018).

63. Walliker, D. et al. Genetic analysis of the human malaria parasite Plasmodium falciparum. Science 236, 1661-1666 (1987).

64. Dankwa, S. et al. Ancient human sialic acid variant restricts an emerging zoonotic malaria parasite. Nat. Commun. 7, 11187 (2016)

65. Hill, D. L., Eriksson, E. M. \& Schofield, L. High yield purification of Plasmodium falciparum merozoites for use in opsonizing antibody assays. J. Vis. Exp. 51590 (2014).

66. Ribaut, C. et al. Concentration and purification by magnetic separation of the erythrocytic stages of all human Plasmodium species. Malar. J. 7, 45 (2008).

67. Johnson, J. D. et al. Assessment and continued validation of the malaria SYBR green I-based fluorescence assay for use in malaria drug screening. Antimicrob. Agents Chemother. 51, 1926-1933 (2007).

68. Wilson, D. W., Crabb, B. S. \& Beeson, J. G. Development of fluorescent Plasmodium falciparum for in vitro growth inhibition assays. Malar. J. 9, 152 (2010).

69. Nguyen, N. et al. Broad activity of diphenyleneiodonium analogues against Mycobacterium tuberculosis, malaria parasites and bacterial pathogens. Eur. J. Med. Chem. 148, 507-518 (2018).

70. Wilson, D. W., Langer, C., Goodman, C. D., McFadden, G. I. \& Beeson, J. G. Defining the timing of action of antimalarial drugs against Plasmodium falciparum. Antimicrob. Agents Chemother. 57, 1455-1467 (2013). 
71. Lin, J. W. et al. Screening inhibitors of P. berghei blood stages using bioluminescent reporter parasites. Methods Mol. Biol. 923, 507-522 (2013).

72. Franke-Fayard, B. et al. A Plasmodium berghei reference line that constitutively expresses GFP at a high level throughout the complete life cycle. Mol. Biochem. Parasitol. 137, 23-33 (2004).

73. Abella, J. V. et al. Met/Hepatocyte growth factor receptor ubiquitination suppresses transformation and is required for Hrs phosphorylation. Mol. Cell Biol. 25, 9632-9645 (2005).

74. Trusolino, L., Bertotti, A. \& Comoglio, P. M. MET signalling: principles and functions in development, organ regeneration and cancer. Nat. Rev. Mol. Cell Biol. 11, 834-848 (2010).

75. Carrolo, M. et al. Hepatocyte growth factor and its receptor are required for malaria infection. Nat. Med. 9, 1363-1369 (2003).

\section{Acknowledgements}

We thank Dr. Kylie Quinn for lending her Flow Cytometry expertise and the staff of Monash FlowCore Facility, in particular Andrew Fryga and Adam Dinsdale, for their continued experimental assistance. We also thank Professor Roger Daly, Dr. Teresa Carvalho, A/Professor Jose Garcia-Bustos and Professor Brian Cooke for fruitful discussions. This work was supported by the Australian Government National Health and Medical Research Council (Project Grants APP1082619 [CD] and APP1143974 [DW]), Australian Research Council PhD Scholarship (AB), Hospital Research Foundation Fellowship (DW) and internal support from Monash University and the University of Adelaide (Beacon Fellowship (DW).

\section{Author contributions}

J.D.A., S.J.F., S.A.J., M.J.B., J.P.S., A.L.B., B.A. and T.M. performed and analysed experiments. J.D.A., S.J.F., D.W.W., O.B. and C.D. designed experiments. J.D.A., D.W.W. and C.D. wrote the manuscript with input from all authors. All the authors approved the manuscript.

\section{Competing interests}

The authors declare no competing interests.

\section{Additional information}

Supplementary information is available for this paper at https://doi.org/10.1038/s41467020-17829-7.

Correspondence and requests for materials should be addressed to C.D

Peer review information Nature Communications thanks David Baker and the other, anonymous, reviewer(s) for their contribution to the peer review of this work. Peer reviewer reports are available.

Reprints and permission information is available at http://www.nature.com/reprints

Publisher's note Springer Nature remains neutral with regard to jurisdictional claims in published maps and institutional affiliations.

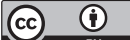

Open Access This article is licensed under a Creative Commons Attribution 4.0 International License, which permits use, sharing, adaptation, distribution and reproduction in any medium or format, as long as you give appropriate credit to the original author(s) and the source, provide a link to the Creative Commons license, and indicate if changes were made. The images or other third party material in this article are included in the article's Creative Commons license, unless indicated otherwise in a credit line to the material. If material is not included in the article's Creative Commons license and your intended use is not permitted by statutory regulation or exceeds the permitted use, you will need to obtain permission directly from the copyright holder. To view a copy of this license, visit http://creativecommons.org/ licenses/by/4.0/.

(C) The Author(s) 2020 